\title{
Discussion on Necessity and Design of AC Filter in Converter Station
}

\author{
Yu Lv \\ North China Electric Power University, Beijing, 102206 \\ alanncepu@foxmail.com
}

Keywords: Harmonic filtering; AC filter; Reactive power compensation; Design method

\begin{abstract}
In today's power grid, harmonics produced by all types of harmonic source will pollute the power system, and massive consumption as well as transmission of reactive power will affect the stability of Electrical equipment operation. It is essential to apply AC Filter, an equipment to reduce harmonics and supply reactive power, to the main source of harmonics and main consumer of reactive power named Converter Station. This article will analyze the reason of applying ACF in convertor station and discuss how to collectively use it and other Reactive power compensation device in order to compensate the reactive power on-site.
\end{abstract}

\section{Introduction}

Harmonics in power system has aroused many people's attention. Meanwhile, the method to reduce it is updating and developing. In today's convertor stations, ACF is widely applied in the AC side, providing harmonics a low impedance path, and thus eliminate the effects of these harmonics.

For the purpose of using ACF to the most extent, it is necessary to learn the mechanism of harmonics' generation, amount of reactive power consumption and working principle of ACF. Also, a flexible adjustment method needs to be obtained. Only in this way can ACF play a full role in power system.

\section{The Generation and Jeopardize of Harmonics in DC Power Engineering}

DC Power Engineering needs convertor station to convert AC/DC power but the convertor itself is the main harmonic source. It is a non-liner component with non-sine current waveform in AC side, containing lots of harmonic component.

Until now, 12-pulsating rectifier inverter units have been widely applied in China's High-Voltage Direct Current project. The circuit is shown in the figure

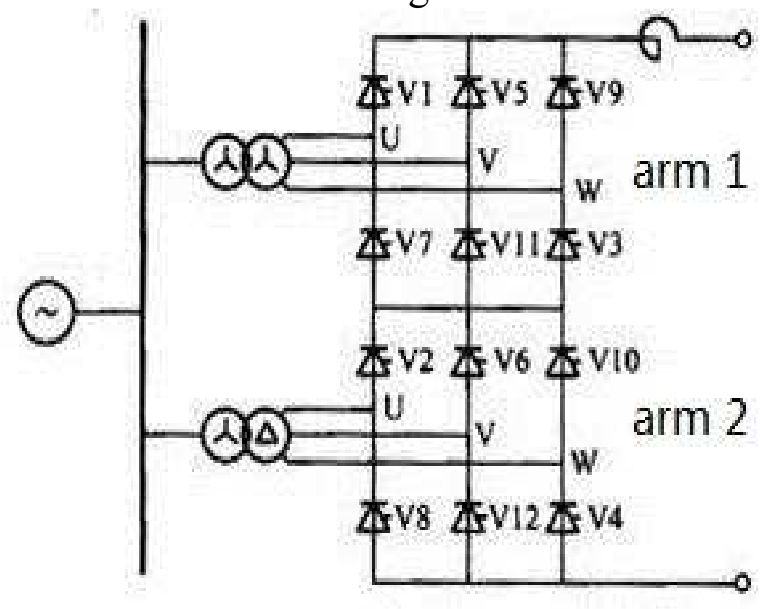

Figure 1. Finite Illustration of 12-pulsating rectifier inverter units

In each power cycle, 12 converter valves take turns to trigger on in order of V1, V2 ... V12, with 30 degree angle interval, and continue to conduct for $120+\mu$ degree $(\mu$ represents commutation angle). Thus the three phase sinusoidal voltage can be converted to 12-pulsating rectified voltage called $\mathrm{U}_{\mathrm{d}}$, and meanwhile direct current Id in DC line can be converted to three phase sinusoidal 
current in AC side.

After Fourier Decomposition, it can be learned that there are 12-interger harmonics in converted voltage and $n$-interger $(n=12 \mathrm{k} \pm 1)$ harmonics in phase current.As a result, 12-pulsating converter becomes a harmonic current source from the perspective of $\mathrm{AC}$ power grid and a harmonic voltage source from the perspective of DC line.

While harmonic current flowing through AC grid, system impedance can cause voltage drop and distortion. Harmonic current and voltage may jeopardize electrical equipment and interfere communication lines nearby. In a word, measures must be taken to ease such dilemma.

\section{The Necessity and Filtering Principle of ACF}

An effective way to reduce harmonics is applying AC Filter to HVDC, which is installed at the AC side of converter station and can provide harmonics a low impedance path to pass through. Because of these functions, ACF is widely used. Here are some types of topology of ACF as shown below.

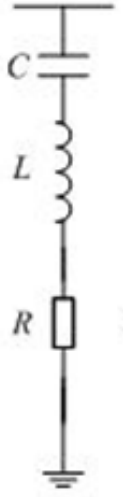

(a)

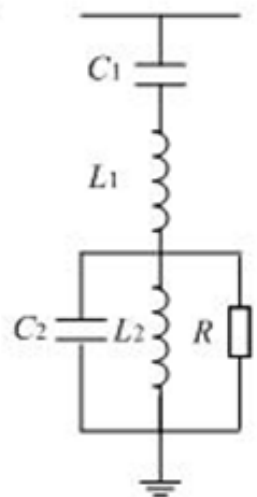

(b)

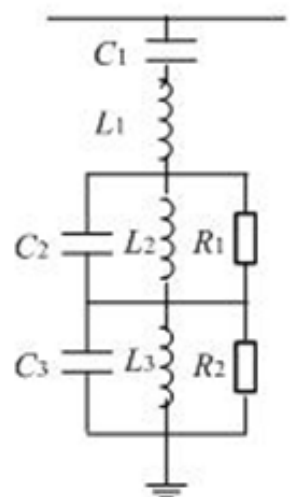

(c)

Figure 2. Finite Topology of ACF

Fig. (a) shows the structure of single tuned filter which has impedance expression for any angular frequency $\omega$. Corresponding $Z-\omega$ curve showed below.

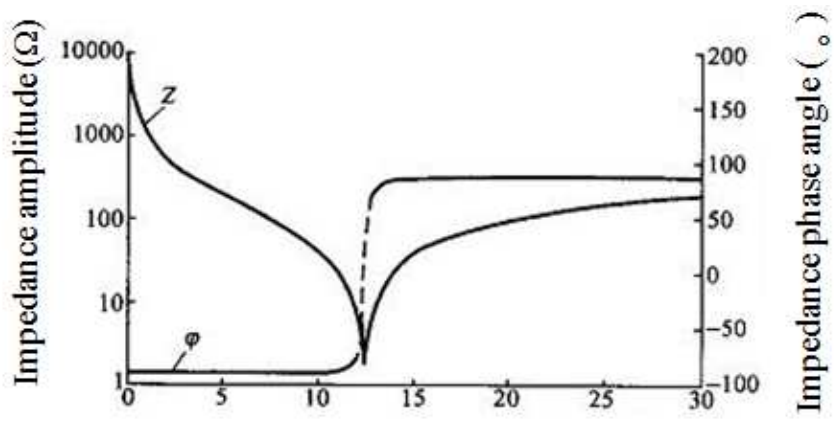

Figure 3. Finite Frequencies of Harmonics

We can learn that single-tuned filter presents a low impedance to harmonic current in a particular frequency, playing the role of shunt. Thus, the amount of harmonic current and voltage flowing to the power system will be declined.

Fig. (b) shows the structure of double-tuned filter. Corresponding $Z-\omega$ curve showed below. 


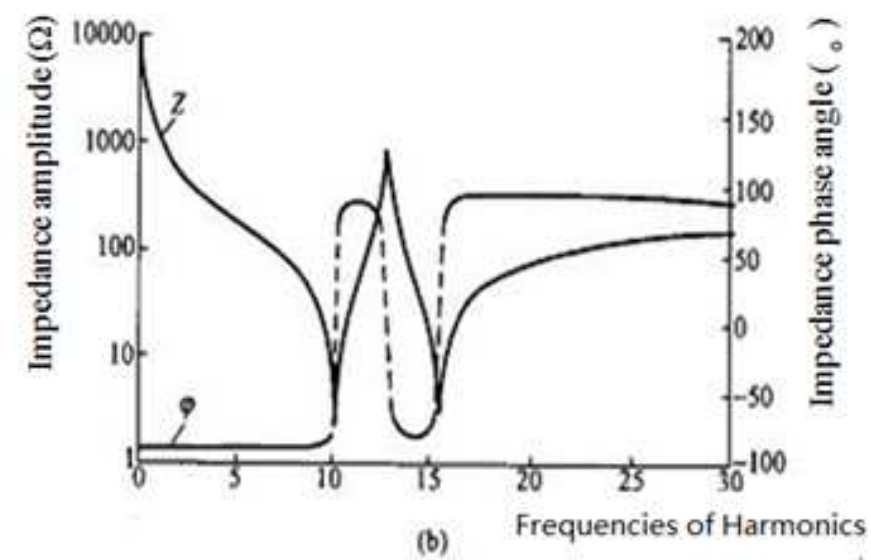

Figure 4. Finite Frequencies of Harmonics

It is clearly presented that double-tuned filter has two specific frequencies orresponding to low impedance, which means it can be applied to eliminatea a wider range of frequencies than single-tuned filter. With this superiority, double-tuned filter is now widely applied in power grid.

Fig. (c) shows the structure of three tuned filter. Similar to double-tuned filter, it presents low impedance to different three frequencies.

\section{Analysis of ACF Design Method}

The understanding of harmonic source and the system it accessing to is fundamental to designing an ACF. The bus voltage distortion rate and harmonic current should meet the requirements while operation of the equipment needs to be safe and economical, and at the same time the grid's status quo, filters' single or total group capacity as well as the environmental conditions ought to be taken into account.

As stated before, there are three main types of ACFs. Single tuned filter has simple structure, low loss, strong ability to suppress single harmonics, and low operation and maintenance requirements. However, tuning harmonics in only one frequency can be a disadvantage which can arise greater investment for more configuration groups. Also balancing reactive power of light loaded ACF is difficult.

Double tuned filter has two specific frequencies orresponding to low impedance. Compared to single tuned filter, it only needs a set of high voltage capacitors $(\mathrm{C} 1)$ and a group of impact reactors(L1), which leads to small investment and low loss. As a result, double tuned filter is now wildely applied in power system.

Three tuned filter is easy to balance reactive power when light loaded and makes investment and loss greatly reduced. However, it is easy to detuning and field tuning is difficult.

For the actual filtering works, determining the filter device scheme needs to depend on the characteristics of the harmonic source. Another two key factors must be taken into account are the capacity and quality of the filter. The larger the capacity of the single group of ACF, the less the total number and investment of filters. But the deviation of the converter bus voltage also comes greater. Thus it is important to design the filter group capacity and the number of groups resonably. Generally, we have the expression of the total number of AC filters $\left({ }^{N_{s}}\right)$ as follows

$$
N_{s} \geq Q_{c} / \Delta Q_{0}
$$

In this inequality, $Q_{c}$ represents the total capacity of ACFs, $\Delta Q_{0}$ represents the single group capacity of ACFs.

The quality of filter indicates the sharpness of the tuning, which increases with the ratio of inductance or capacitance to resistance increasing. The aim of choosing the quality of filter is to achieve optimized operation of ACFs. ACFs with high value of Q can reduce the filter loss and harmonic voltage when tuned correctly but will increase the risk of parallel resonance between the 
filter and the network. So the value of Q should be chosen based on the actual situation.

\section{Cooperation of ACF and Reactive Power Compensation}

HVDC converter station, using thyristor converter valve for commutation and grid power phase commutation control technology, absorbs a great deal of reactive power which is proportional to the active power exchanged between the converter station and the AC system. So large amount of reactive power may cause the grid to collapse.

Converter needs the reactive power when operating but the reactive power can't be mainly dependent on exchanges system to provide. So there is an urgent to install the reactive power compensation device.

In order to meet the change in DC power, the reactive power compensation device must be grouped and switched. It is usually divided into $2 \sim 4$ groups and in every group there are $2 \sim 4$ groups of reactive power compensation device. Various types of reactive power compensation devices need to be distributed evenly among the reactive groups as much as possible.

Here are the principles of determining the group capacity. (1) Considering the total reactive power compensation capacity of converter station, the effect of switching reactive power compensation device, AC/DC system voltage control capability, ACF's performance and the position of the reactive power compensation device. (2) Should meet the exchange of transient voltage change rate and steady-state voltage requirements. (3) Avoid resonance with adjacent synchronous motors. (4) Any group of switching can't cause the inverter commutation failure.

As the AC filter investment is higher than the same capacity of the parallel capacitor group, and in the case of performance requirements and converter station reactive power balance, the number of AC filter banks should be as small as possible. The reactive power shortage section is compensated using a shunt capacitor.

In order to maintain the station inductive reactive power balance and in addition to increasing the trigger angle to increase the converter station reactive power consumption, if necessary, a separate inductive reactive power compensation device is needed to installed. In the case of filtering requirements, the capacity of a single $\mathrm{AC}$ filter should be minimized as much as possible.

In order to improve the economics of $\mathrm{DC} / \mathrm{AC}$ station reactive power compensation device program, if necessary, consider the $\mathrm{AC}$ filter and parallel capacitor group to take different packet capacity configuration program. From the point of view of reducing the potential of the equipment investment and the low power level of the inverter, the AC filter and the capacitor group in the converter station adopt the different packet capacity configuration scheme and the AC filter capacity should take a relatively low value, the capacitor group Capacity may be appropriate.

\section{References}

[1] Han Minxiao, Principle and Operation of HVDC Transmission [M].Beijing: Machinery Industry Press, 2012:101-147.

[2] Li Xingyuan. HVDC system [M].Beijing: Science Press, 2010:43-56.

[3] Wang Zhao'an. Harmonic suppression and reactive power compensation [M].Beijing: Machinery Industry Press, 2015:106-111.

[4] Wen Jun. Optimum Design of AC Filter for $\pm 800 \mathrm{KV}$ UHVDC Transmission Project [J]. High voltage technology .2010.36(4):912-917.

[5] Chou C J, Liu C W, Lee J Y, et al. Optimal planning of large passive-harmonic[J]. Power Systems IEEE Transactions on, 2000, 15(1):433-441.

[6] Yin W, Yang Z. Study on Reactive Power Balance and Compensation Strategy for UHVDC Project [J]. High Voltage Engineering, 2006, 32(9):50-54.

[7] Arrillaga J. High Voltage Direct Current Transmission [M]. 1998.

[8] Chen X, Han M, Liu C, et al. Modeling of a Large Scale UHVAC/DC Power Networks Based on PSCAD/EMTDC[C]// Power and Energy Engineering Conference. IEEE, 2010:1-4. 
[9] Long J, Han M, Guo X, et al. Strategy of Emergency Power Support and Voltage Stability Control with HVDCs[J]. Modern Electric Power, 2010.

[10]http://baike.so.com/doc/4391799-4598440.html. 